\section{Inadequate Knowledge and Practice Regarding Complementary Feeding in Mothers from South Asian Countries - Need for Global Public Health Intervention Program}

Received: December 22, 2016; Accepted: December 23, 2016; Published: December 26, 2016

World Health Organization (WHO)/United Nations Children's Fund (UNICEF) emphasizes that optimum nutrition is a must for children especially during the first 1000 days of life ( 270 days in-utero and 2 years after birth) as maximal growth and development occurs during this period and suboptimal nutrition during this period can cause significant growth and development retardation. WHO recommends exclusive breastfeeding in infants for $1^{\text {st }}$ six months of life and introduction of complementary feeds from 6-24 months with continuation of breastfeeding for a period of 2 years and beyond? Complementary feeds (CF) are introduced at 6 months as breast milk alone is no longer sufficient to meet the nutritional requirements of infants, and therefore other foods and liquids are needed, along with breast milk to meet the nutritional needs of the child $[1,2]$.

WHO, UNICEF, and many other international and national agencies have described detailed guidelines regarding the composition, consistency, safety and feeding practices for Complementary Feeding (CF). However, the practical knowledge and understanding in mothers regarding CF seems to be limited especially in mothers from South Asian countries.

In India, in study to access knowledge and attitude of mothers regarding CF in Indore and Udaipur city it was observed that $14 \%$ mothers did not initiate CF even after 6 months [3,4]. Quantity of CF given by the mothers was found to be insufficient with only $32 \%$ mothers giving adequate complementary feeds in South Coastal India [5]. Consistency of CF was found to be very thin in $59 \%$ mothers [3]. Biscuits were preferred as complementary food by $32-64 \%, 50-52 \%$ mothers preferred thin pulsel water/rice water, $62 \%$ preferred commercial food formula as CF indicating that mothers were unaware about the health benefits of home cooked CF and appropriate consistency of CF in India $[3,4]$.

In a study conducted in $\mathbf{4 0 0}$ mothers visiting a hospital in Dhaka, Bangladesh it was found that $51.3 \%$ mother initiated CF early, type of $1^{\text {st }} \mathrm{CF}$ was inappropriate in $72.9 \%$ mother and $43.8 \%$ had inappropriate frequency of CF [6]. Rice gruel with milk was the $1^{\text {st }} \mathrm{CF}$ used by $26.56 \%$ mothers indicating very early initiation of animal milk in the diet of infants. In another study, it was found that only $11.3 \%$ mothers washed hands and utensils properly

\section{Neha Sanwalka \\ Department of Nutrition and Biostatistics, Nutri Canvas, Mumbai, India}

Corresponding author:

Neha Sanwalka

neha.sanwalka@gmail.com

Department of Nutrition and Biostatistics, Nutri Canvas, Mumbai, India.

Tel: 91-8108009900

Citation: Sanwalka N. Inadequate Knowledge and Practice Regarding Complementary Feeding in Mothers from South Asian Countries - Need for Global Public Health Intervention Program. J Clin Nutr Diet. 2016, 2:4.

before cooking CF where $76 \%$ only washed hands properly and another $5.6 \%$ only washed utensils properly indicating inadequate knowledge regarding hygiene for preparing CF in mothers [7].

In a cross sectional study done in mothers visiting paediatric department of a hospital in Pakistan, it was found that the correct knowledge of initiation of CF was in 54\% of mothers but it was practiced by only $43 \%$. The overall knowledge regarding CF was good in $24 \%$ mothers, $28 \%$ had poor knowledge. Forty eight mothers gave incorrect consistency of CF, 51\% gave the correct amount of CF whereas only $7 \%$ women had overall good practices for CF [8]. In another study, it was found that $71.7 \%$ mothers did not boil drinking water whereas $12.3 \%$ mothers gave tea and crackers as first solid foods to their infants in Pakistan [9].

In South Asian country of Nepal, about half mothers fed CF of appropriate consistency where as $60 \%$ fed the correct amounts of CF to their infants, $33.27 \%$ used appropriate frequency to feed CF whereas $9.9 \%$ offered CF to infants more frequently that recommended [10]. In a hospital based study from Sri Lanka, similar results were obtained with $48 \%$ mothers giving satisfactory CF, $17 \%$ mothers giving satisfactory frequency but unsatisfactory quality of $C F, 9 \%$ mothers giving satisfactory quality but unsatisfactory frequency of CF and $26 \%$ giving unsatisfactory CF [11].

Studies on mothers from all 5 South Asian countries: India, Bangladesh, Nepal, Pakistan and Sri Lanka highlighted the lack 
of adequate knowledge regarding CF in mothers. Knowledge regarding almost all aspects of appropriate CF were lacking in mothers from all countries. A big proportion of mothers from these countries fed thin $\mathrm{CF}$, had inappropriate frequency of feeding CF, chooses wrong first foods or did not maintain adequate hygiene while preparing CF [3-11]. Feeding inadequate and inappropriate CF or delayed CF can lead to energy deficit, delayed growth, increase risk for malnutrition and cause anaemia in infants [1]. Too early introduction can displace mother's milk and benefits associated with breast feeding [1]. Hence it is very important to plan global public health policies to promote correct CF practices in mothers. It is important that steps are taken to impart knowledge regarding correct infant young and child feeding practices to mothers. Unless WHO/UNICEF or other national guidelines are conveyed to mothers through public health programs, there is likelihood of CF practices remaining poor in developing countries. Hence, it is very essential to plan ways to teach mothers about correct CF. 


\section{References}

1 http://www.who.int/nutrition/publications/guiding_principles_ compfeeding_breastfed.pdf

2 Tiwari S, Bharadva K, Yadav B, Malik S, Gangal P, et al. (2016) Infant And Young Child Feeding Guidelines. Indian Pediatr 53: 703-713.

3 Lodha S, Bharti V (2013) Assessment of complementary feeding practices and misconceptions regarding foods in young mothers. Int J Food Nutr Sci 2: 85-90.

4 Bhatia R, Jain U (2014) Knowledge, attitude, practices and misconceptions among mothers regarding complementary feeding. Int J Med Sci Public Health 3: 1251-1253.

5 Rao S, Swathi PM, Unnikrihnan B, Hegde A (2011) Study of complementary feeding practices among mothers of children aged six months to two years - a study from coastal south India. Aus Med J 4: 252-257.

6 Paul Sk, Islam QR, Roy S, Rudra PK (2014) Complimentary feeding practices in under 2 children. Chattagram Maa-O-Shishu Hospital Medical College Journal 13: 35-41.
7 Sultana S, Hoque A, Saleh F (2014) Infant and young child feeding practices and their nutritional status in National Nutrition Programmer Area in Bangladesh: A cross sectional study. J Hum Nutr Food Sci 2: 1028-1033.

8 Hasnain S, Majrooh MA, Anjum R (2013) Knowledge and Practices of Mothers For Complementary Feeding In Babies Visiting Pediatrics Outpatient Department Of Jinnah Hospital, Lahore. Biomedica 29: 221-230.

9 Mohsin SS, Shaikh AS, Shaikh R, Haider N, Parkash A (2014) Knowledge attituce and practices of mothers regarding complementary feeding. Journal of Dow University of Health Sciences Karachi 8: 21-25.

10 Chapagain RH (2013) Complementary feeding practices of Nepali mothers for 6 months to 24 months children. J Nepal Med Assoc 52: 443-448.

11 Danasuriya MN, Guruge K, Wijewardena RRK (2013) A hospital based study on the usage pattern of Child Health Development Record as an information source on infant and young child feeding. Sri Lanka Journal of Child Health 42: 133-138. 